\title{
Electronic structure study of double perovskites
}

\author{
$A_{2} \mathrm{FeReO}_{6}(A=\mathrm{Ba}, \mathrm{Sr}, \mathrm{Ca})$ and $\mathrm{Sr}_{2} M \mathrm{MoO}_{6}$ \\ $(M=\mathrm{Cr}, \mathrm{Mn}, \mathrm{Fe}, \mathrm{Co})$ by LSDA and LSDA $+U$
}

\author{
Hua $\mathrm{Wu}$ \\ Max-Planck-Institut für Physik komplexer Systeme, D-01187 Dresden, Germany \\ and Institute of Solid State Physics, Academia Sinica, 230031 Hefei, P. R. China
}

\begin{abstract}
We have implemented a systematic LSDA and LSDA $+U$ study of the double perovskites $A_{2} \mathrm{FeReO}_{6} \quad(A=\mathrm{Ba}, \mathrm{Sr}, \mathrm{Ca})$ and $\mathrm{Sr}_{2} M \mathrm{MoO}_{6}$ $(M=\mathrm{Cr}, \mathrm{Mn}, \mathrm{Fe}, \mathrm{Co})$ for understanding of their intriguing electronic and magnetic properties. The results suggest a ferrimagnetic (FiM) and half-metallic (HM) state of $A_{2} \mathrm{FeReO}_{6}(A=\mathrm{Ba}, \mathrm{Sr})$ due to a $p d d-\pi$ coupling between the down-spin $\mathrm{Re}^{5+} / \mathrm{Fe}^{3+} t_{2 g}$ orbitals via the intermediate $\mathrm{O} 2 p_{\pi}$ ones, also a very similar $\mathrm{FiM}$ and $\mathrm{HM}$ state of $\mathrm{Sr}_{2} \mathrm{FeMoO}_{6}$. In contrast, a decreasing Fe $t_{2 g}$ component at Fermi level $\left(E_{F}\right)$ in the distorted $\mathrm{Ca}_{2} \mathrm{FeReO}_{6}$ partly accounts for its nonmetallic behavior, while a finite $p d d-\sigma$ coupling between the down-spin $\mathrm{Re}^{5+} / \mathrm{Fe}^{3+} e_{g}$ orbitals being present at $E_{F}$ serves to stabilize its FiM state. For $\mathrm{Sr}_{2} \mathrm{CrMoO}_{6}$ compared with $\mathrm{Sr}_{2} \mathrm{FeMoO}_{6}$, the coupling between the down-spin $\mathrm{Mo}^{5+} / \mathrm{Cr}^{3+} t_{2 g}$ orbitals decreases as a noticeable shift
\end{abstract}


up of the $\mathrm{Cr}^{3+} 3 d$ levels, which is likely responsible for the decreasing $T_{\mathrm{C}}$ value and weak conductivity. Moreover, the calculated level distributions indicate a $\mathrm{Mn}^{2+}\left(\mathrm{Co}^{2+}\right) / \mathrm{Mo}^{6+}$ ionic state in $\mathrm{Sr}_{2} \mathrm{MnMoO}_{6}\left(\mathrm{Sr}_{2} \mathrm{CoMoO}_{6}\right)$, in terms of which their antiferromagnetic insulating ground state can be interpreted. While orbital population analyses show that owing to strong intrinsic $p d$ covalence effects, $\mathrm{Sr}_{2} M \mathrm{MoO}_{6}(M=\mathrm{Cr}, \mathrm{Mn}, \mathrm{Fe}, \mathrm{Co})$ have nearly the same valence state combinations, as accounts for the similar $M$-independent spectral features observed in them.

PACS numbers: 71.20.-b, 75.50.-y, 71.15.Mb 


\section{INTRODUCTION}

A recent finding of the room-temperature tunnelling magnetoresistance (TMR) effect in double perovskites $\mathrm{Sr}_{2} \mathrm{FeMoO}_{6}$ ( $\mathrm{SFMO}$ ) and $\mathrm{Sr}_{2} \mathrm{FeReO}_{6}$ (SFRO) ? $^{2}$ revives the study of $3 d(M)$ and $4 d / 5 d\left(M^{\prime}\right)$ transition-metal oxide alloys $A_{2} M M^{\prime} \mathrm{O}_{6}(A=\mathrm{Ba}, \mathrm{Sr}, \mathrm{Ca})$. Most of the alloys have been up to now found to take a rock-salt crystal structure, an ordered one of the alternate perovskite units $A M \mathrm{O}_{3}$ and $A M^{\prime} \mathrm{O}_{3}$ along three crystallographical axes. SFMO and SFRO taking this structure are predicted by band calculations to be a HM, where energy bands of one spin channel (up or down) cross $E_{F}$ and are therefore metallic but those of the other spin channel are separated by an insulating gap. Theoretically speaking, the conduction electrons are $100 \%$ spin polarized, this effect generally related to a ferromagnetic (FM) ordering of a (sub)lattice, despite a possible inter-sublattice antiferromagnetic (AFM) coupling in some cases. For the polycrystallic ceramics of SFMO and SFRO, an applied magnetic field can control magnetic domain reorientation and tends to parallelize the magnetization directions of FM domains and therefore reduces the spin scattering of spin-polarized carriers at grain boundaries, giving rise to a significant decrease of the measured resistivity.

TMR. Like the well-known HM-like colossal MR manganites, 1 the TMR materials also have potential technological applications to magnetic memory and actuators.

The MR materials with an interesting combination of electronic and magnetic properties are of current considerable interest also from the basic points of view. Double exchange (DE) l $^{0}$ model has been widely employed to account for the FM metallicity of $\mathrm{La}_{1-x} \mathrm{Ca}_{x} \mathrm{MnO}_{3}$ and related compounds, in spite of its recent modification by taking into account a strong electron-phonon coupling arising from the Jahn-Teller splitting of the 
$\mathrm{Mn}^{3+}$ ions, 6 and even an alternative $p$ - $d$ exchange model as lately suggested for those O $2 p$ - $M 3 d$ charge-transfer oxides.6 One DE-like mechanism 89 was suggested for SFMO [SFRO] that a strong hybridization between the $\mathrm{Mo}^{5+}\left(4 d^{1}\right)\left[\mathrm{Re}^{5+}\left(5 d^{2}\right)\right]$ and $\mathrm{Fe}^{3+}\left(3 d^{5}\right)$ down-spin $t_{2 g}$ orbitals via a $p d d-\pi$ coupling leads to electron mobility being responsible for the HM behavior, and the itinerant down-spin carriers are antiferromagnetically polarized by the localized $\mathrm{Fe}^{3+} S=5 / 2$ full up-spins and therefore mediate a FM coupling in the Fe sublattice. Alternatively, very late a new mechanism 910 was proposed that if the Fe sublattice has a FM ordering (being so actually), a bonding-antibonding splitting due to the Mo $4 d[\operatorname{Re} 5 d] /$ Fe $3 d$ hybridization results in a shift up (down) of the Mo $4 d[\operatorname{Re} 5 d]$ $t_{2 g}^{\uparrow}\left(t_{2 g}^{\downarrow}\right)$ bands located between the $\mathrm{Fe}^{3+} 3 d$ full-filled $t_{2 g}^{\uparrow}$ and empty $t_{2 g}^{\downarrow}$ bands, and therefore an electron transfer from Mo [Re] $t_{2 g}^{\uparrow}$ bands to $t_{2 g}^{\downarrow}$ ones. This energy gain causes a negative spin polarization of the formally nonmagnetic Mo [Re] species, and the itinerant electrons in the partly filled $t_{2 g}^{\downarrow}$ conduction bands cause kinetic energy gain further via the DE interaction, both of which commonly stabilize the FM state, as compared with the AFM or paramagnetic (PM) state.

In contrast, it was argued in another late study 11 that a direct Re $t_{2 g}$-Re $t_{2 g}$ interaction is the main cause for the metallic behavior of $\mathrm{Ba}_{2} \mathrm{FeReO}_{6}(\mathrm{BFRO})$, and a lattice distortion of $\mathrm{Ca}_{2} \mathrm{FeReO}_{6}$ (CFRO) induced by the smaller-size Ca species disrupts the Re-Re interaction and makes itself nonmetallic. While magnetization measurements show that the latter has a higher $T_{\mathrm{C}}$ value than the former, which seems surprising when referring to their distinct conduction behaviors.11

One of the aims of this work is to probe which mechanism, either the direct or the indirect Re-Re interaction is essentially responsible for the conduction behavior of BFRO and SFRO by means of density functional theory (DFT) 12 calculations. The present 
results clearly show that the Re-O-Fe-O-Re interaction rather than the direct Re-Re one takes the responsibility. And it is suggested, based on the calculated orbital-resolved density of states (DOS), that a reduced Re $t_{2 g}$-Fe $t_{2 g} p d d-\pi$ hybridization in distorted CFRO partly accounts for its nonmetallic behavior, compared with the cases of cubic BFRO and SFRO. While a presence of a finite Re $e_{g}$-Fe $e_{g} p d d-\sigma$ hybridization in CFRO at $E_{F}$ contributes to its increasing $T_{\mathrm{C}}$.

On the other hand, the dependence of the electronic, magnetic, and transport properties of $\mathrm{Sr}_{2} M \mathrm{MoO}_{6}(M=\mathrm{Cr}, \mathrm{Mn}, \mathrm{Fe}, \mathrm{Co})$ on the $3 d$ transition-metal species is an intriguing issue. $812 \mathrm{SFMO}$ and $\mathrm{Sr}_{2} \mathrm{CrMoO}_{6}$ have a high $T_{\mathrm{C}}$ value, and the former is a metallic compound while the latter is a nonmetal with a relatively low room-temperature resistivity. 8 In contrast, $\mathrm{Sr}_{2} \mathrm{MnMoO}_{6}$ and $\mathrm{Sr}_{2} \mathrm{CoMoO}_{6}$ are AFM insulators. 13 For the reason, DFT calculations are also designed for them. The valence states, spin moments, electronic and magnetic characters are discussed below in detail.

\section{COMPUTATIONAL DETAILS}

The structure data of $A_{2} \mathrm{FeReO}_{6}(A=\mathrm{Ba}, \mathrm{Sr}, \mathrm{Ca})$ and $\mathrm{Sr}_{2} M \mathrm{MoO}_{6}(M=\mathrm{Cr}, \mathrm{Mn}, \mathrm{Fe}, \mathrm{Co})$ are taken from Refs. 11, 2 and 8. These compounds have a cubic crystal structure except for the tetragonal $\mathrm{Sr}_{2} \mathrm{CoMoO}_{6}$ and monoclinic CFRO. An orthogonal cell is assumed for CFRO in order to simplify calculations, since the experimentally determined monoclinic cell $(\beta=90.02)$ deviates slightly from an orthogonal one 11 FM calculations are performed for these compounds. As seen below, there is an induced negative spin polarization of the Re or Mo sublattice at the background of the FM Fe and/or Cr sublattices, which we call a FiM structure in this paper. In addition, the present FM solutions of $\mathrm{Sr}_{2} \mathrm{MnMoO}_{6}$ and 
$\mathrm{Sr}_{2} \mathrm{CoMoO}_{6}$ can be extended to an explanation of their AFM insulating behaviors.

The full-potential linearly combined atomic-orbital (LCAO) band method 14 based on the local spin density approximation (LSDA) 12 to DFT and on-site Coulomb correlation correction $(\mathrm{LSDA}+U)^{15}$, is adopted in the present calculations. Hartree potential is expanded in terms of lattice harmonics up to $L=6$, and an exchange-correlation potential of von Barth-Hedin type 16 is adopted. The $U(=3,4,4.5,5 \mathrm{eV})$ parameters are used for the strongly correlated $M(=\mathrm{Cr}, \mathrm{Mn}, \mathrm{Fe}, \mathrm{Co}) 3 d$ electrons, 17 respectively; while a small $U=1 \mathrm{eV}$ for the weakly correlated Re $5 d$ and Mo $4 d$ electrons. 18 Ba $5 p 5 d 6 s / \mathrm{Sr} 4 p 4 d 5 s / \mathrm{Ca} 3 p 3 d 4 s$, $M 3 d 4 s$, Re $5 d 6 s / \mathrm{Mo} 4 d 5 s$, and O 2s2p orbitals are treated as valence states. 125 (64 for CFRO with a doubled cell) special $k$ points in irreducible Brillouin zone are used in the present self-consistent calculations.

A description of the method for orbital population analyses is given below for the reason that a detailed discussion about the orbital occupation is made in the text.

A crystal wave function $\Psi_{j}^{\mathbf{k}}$ is expressed in terms of Bloch basis functions $\left\{\Phi_{l}^{\mathbf{k}}\right\}$ in the LCAO formalism:

$$
\Psi_{j}^{\mathbf{k}}=\sum_{l} C_{l}^{\mathbf{k} j} \Phi_{l}^{\mathbf{k}}=\sum_{l} C_{l}^{\mathbf{k} j} \frac{1}{\sqrt{N}} \sum_{m} e^{i \mathbf{k} \cdot\left(\tau+\mathbf{R}_{m}\right)} \phi_{l}\left(\mathbf{r}-\tau-\mathbf{R}_{m}\right)
$$

where $\phi_{l}$ denotes an atomic orbital wave function. $\mathbf{k}, j, l, N, \mathbf{R}_{m}$, and $\tau$ stand for, respectively, a wave vector, a band index, an atomic orbital label, number of unit cells, a lattice vector, and an atomic position.

The total DOS reads

$$
\begin{aligned}
\rho(E) & =\frac{1}{N_{\mathbf{k}}} \sum_{\mathbf{k} j}\left|\Psi_{j}^{\mathbf{k}}\right|^{2} \delta\left(E-E_{j}^{\mathbf{k}}\right)=\frac{1}{N_{\mathbf{k}}} \sum_{\mathbf{k} j} \sum_{l l^{\prime}} C_{l}^{\mathbf{k} j} C_{l^{\prime}}^{\mathbf{k} j *} S_{l^{\prime} l}^{\mathbf{k}} \delta\left(E-E_{j}^{\mathbf{k}}\right) \\
& =\frac{1}{N_{\mathbf{k}} N} \sum_{\mathbf{k} j} \sum_{l m l^{\prime} m^{\prime}} C_{l}^{\mathbf{k} j} C_{l^{\prime}}^{\mathbf{k} j *}<\phi_{l^{\prime}} \mid \phi_{l}>e^{i \mathbf{k} \cdot\left(\tau+\mathbf{R}_{m}-\tau^{\prime}-\mathbf{R}_{m^{\prime}}\right)} \delta\left(E-E_{j}^{\mathbf{k}}\right),
\end{aligned}
$$

where 


$$
S_{l^{\prime} l}^{\mathbf{k}}=<\Phi_{l^{\prime}}^{\mathbf{k}} \mid \Phi_{l}^{\mathbf{k}}>
$$

refers to an overlap matrix, and $N_{\mathbf{k}}$ represents the number of $\mathbf{k}$-points.

$\rho(E)$ can be decomposed into an on-site term $\rho_{A}(E)\left(m=m^{\prime}\right.$ and $\left.\tau=\tau^{\prime}\right)$ and an overlap term $\rho_{B}(E)\left(m \neq m^{\prime}\right.$ or $\left.\tau \neq \tau^{\prime}\right)$. The on-site term is written as

$$
\rho_{A}(E)=\frac{1}{N_{\mathbf{k}}} \sum_{\mathbf{k} j} \sum_{l}\left|C_{l}^{\mathbf{k} j}\right|^{2} \delta\left(E-E_{j}^{\mathbf{k}}\right)
$$

due to the orthogonality and normality of atomic orbitals at the same site. Correspondingly, the on-site occupation number $Q_{A}$

$$
Q_{A}=\int \rho_{A}(E) d E=\frac{1}{N_{\mathbf{k}}} \sum_{\mathbf{k} j} \sum_{l}\left|C_{l}^{\mathbf{k} j}\right|^{2}
$$

is composed of atomic contributions $\left\{\left|C_{l}^{\mathbf{k} j}\right|^{2}\right\}$. Obviously, the sum rule for total number of electrons is not satisfied due to the non-orthogonality of atomic orbitals at different sites. For the reason, the overlap term $\rho_{B}(E)$ is dealt with by Mulliken analysis 19 which is widely used among quantum chemistry community and provides a reasonable description of local orbitals.

The overlap term is expressed as

$$
\begin{aligned}
\rho_{B}(E) & =\frac{1}{N_{\mathbf{k}}} \sum_{\mathbf{k} j} \sum_{l, l^{\prime} \neq l} C_{l}^{\mathbf{k} j} C_{l^{\prime}}^{\mathbf{k} j *} S_{l^{\prime} l}^{\mathbf{k}} \delta\left(E-E_{j}^{\mathbf{k}}\right) \\
& =\frac{1}{N_{\mathbf{k}}} \sum_{\mathbf{k} j} \sum_{l} \sum_{l^{\prime} \neq l} \frac{\left|C_{l}^{\mathbf{k} j}\right|^{2}}{\left|C_{l}^{\mathbf{k} j}\right|^{2}+\left|C_{l^{\prime}}^{\mathbf{k} j}\right|^{2}}\left(C_{l}^{\mathbf{k} j} C_{l^{\prime}}^{\mathbf{k} j *} S_{l^{\prime} l}^{\mathbf{k}}+C_{l}^{\mathbf{k} j *} C_{l^{\prime}}^{\mathbf{k} j} S_{l l^{\prime}}^{\mathbf{k}}\right) \delta\left(E-E_{j}^{\mathbf{k}}\right) \\
& =\frac{1}{N_{\mathbf{k}}} \sum_{\mathbf{k} j} \sum_{l} D_{l}^{\mathbf{k} j} \delta\left(E-E_{j}^{\mathbf{k}}\right) .
\end{aligned}
$$

Mulliken analysis is taken such way that the overlap term is decomposed into atomiclike contributions $D_{l}^{\mathbf{k} j}$, according to their respective 'weight' $\left|C_{l}^{\mathbf{k} j}\right|^{2}$.

As a result, one can calculate the orbital-resolved DOS and population, respectively, by the expressions 


$$
\begin{gathered}
\rho(E)=\rho_{A}(E)+\rho_{B}(E)=\frac{1}{N_{\mathbf{k}}} \sum_{\mathbf{k} j} \sum_{l}\left(\left|C_{l}^{\mathbf{k} j}\right|^{2}+D_{l}^{\mathbf{k} j}\right) \delta\left(E-E_{j}^{\mathbf{k}}\right), \\
Q=\frac{1}{N_{\mathbf{k}}} \sum_{\mathbf{k} j} \sum_{l}\left(\left|C_{l}^{\mathbf{k} j}\right|^{2}+D_{l}^{\mathbf{k} j}\right) .
\end{gathered}
$$

Note that for a spin-polarized system, one can calculate the contributions from up- and down-spin channels and then obtain the values of spin moments.

\section{RESULTS AND DISCUSSIONS}

The LSDA calculations for BFRO and SFRO give a nearly same HM solution as recently reported for the latter.10 Their level distributions are overall very similar, as is not surprising since, on one hand, both compounds have the same cubic structure except for a difference in their lattice constants $8.05 \AA$ versus $7.89 \AA$; 11 . $\mathrm{Ba}^{2+} / \mathrm{Sr}^{2+}$ ions per formula unit donate their four valence electrons, [all $A(=\mathrm{Ba}, \mathrm{Sr}, \mathrm{Ca})$ almost taking a valence state of +1.8 and a minor spin moment of $-0.01 \mu_{B}$ in the present calculations], into $\left(\mathrm{FeReO}_{6}\right)^{-4}$ unit and scarcely affect the valence bond interactions both in and between the $\left(\mathrm{FeReO}_{6}\right)^{-4}$ units. Owing to the lattice distortion of $\mathrm{CFRO}$ - shrinked Fe-O-Re bond lengths and bent bond angles1 _ and therefore modified $p d d$ hybridizations, however, a noticeable difference emerging in CFRO is that the down-spin $e_{g}$ hybridized states are also present at $E_{F}$. It can be seen in Fig. 1 that in $A_{2} \mathrm{FeReO}_{6}(A=\mathrm{Ba}, \mathrm{Sr}, \mathrm{Ca})$, the up-spin Fe $3 d$ orbitals are almost full-filled, while the down-spin Fe $3 d$ ones are nearly empty except for a small amount of occupations due to $p d$ hybridizations, both of which indicate that the formal $\mathrm{Fe}^{3+}\left(3 d^{5}\right)$ ions actually take a mixing $3 d^{5}+3 d^{6} L$ state $(L$ : a ligand hole) like an ordinary case, as also seen in Table I. Moreover, a small increase of Fe $3 d$ population (and a corresponding minor decrease of Fe spin) as the change of 
$A$ from $\mathrm{Ca}$ via $\mathrm{Sr}$ to $\mathrm{Ba}$, being qualitatively in accord with the Mössbauer spectroscopy study 11 indicates a decreasing oxidization of Fe, which is also supported by the calculated valence state of the coordinated oxygens, -1.47 for $A=\mathrm{Ca},-1.4$ for $A=\mathrm{Sr}$, and -1.35 for $A=$ Ba. While a significantly strong $p d$ hybridization effect is evident for the formal high valence $\operatorname{Re}^{5+}$ ions, which causes a larger bonding-antibonding splitting and a crystal-field $t_{2 g}-e_{g}$ splitting. As a result, although the down-spin Re $t_{2 g}$ states crossing $E_{F}$ are partly occupied as the expected $2 / 3$ filling, the $5 d$ orbital population is larger than 4 and far away from the formal $\mathrm{Re}^{5+} 5 d^{2}$ as seen in Table I. A similar population difference larger than 2 also appears in the high valence oxides, e.g., $\mathrm{V}_{2} \mathrm{O}_{5} 20$ and $\mathrm{NaV}_{2} \mathrm{O}_{5} .21$ The relatively smaller Re $5 d$ population in CFRO, compared with SFRO and BFRO, is also related to the relatively higher negative valence of the coordinated oxygens. A stronger ionicity of CFRO could be implied by a weaker Fe-O-Re covalence interaction caused by the bent Fe-O-Re bond angle. Moreover, a little smaller Re $5 d$ population in SFRO than in BFRO could be due to a more delocalized behavior of the down-spin Re $t_{2 g}$ orbitals in SFRO, which is closely related to a larger band width caused by the smaller cubic lattice constant of SFRO.

As seen in Fig. 1, the Re $t_{2 g}$ levels exactly lie between the up- and down-spin Fe $t_{2 g}$ ones, and they are close to the down-spin Fe $t_{2 g}$ levels. As a result, the bondingantibonding mechanism stated above induces a spin splitting of the Re $5 d$ levels and a subsequent electron transfer and therefore a negative spin polarization. Moreover, it is evident that a stronger Re/Fe bonding-antibonding interaction occurs in the down-spin channel than in the up-spin one. The present LSDA calculations give the Fe (Re) spin moments of $3.72(-0.99) \mu_{B}$ in BFRO, and $3.75(-1.05) \mu_{B}$ in SFRO, and $3.76(-1.07) \mu_{B}$ in CFRO, all of which are reduced by the $p d$ hybridizations, compared with the ideal 5 (- 
2) $\mu_{B}$ for $\mathrm{Fe}^{3+}\left(\mathrm{Re}^{5+}\right)$. A stronger Re $5 d$ spin polarization in SFRO than in BFRO could be due to a stronger bonding-antibonding mechanism in SFRO caused by the shorter Fe-O-Re bond. While the calculated largest Re spin in CFRO is ascribed to both an additional negative spin polarization of the Re $e_{g}$ electrons and weaker delocalization of the Re $5 d$ electrons. The calculated total spin, including both the weakly polarized oxygen spins with an averaged value of $0.05 \mu_{B}$ and the minor $A$ spin, is accurately equal to $3 \mu_{B}$ per formula unit as an expected integral value for a HM. While the reduced experimental values, e.g. $2.7 \mu_{B}$ in $\mathrm{Sr}_{2} \mathrm{FeReO}_{6}$, 2 are probably ascribed to a site-disorder effect as previously suggested.122 The calculated Fe spin moments agree well with the previous $3.7 \mu_{B}$ in SFRO 10 while the Re spin moments are larger than the corresponding value of $-0.78 \mu_{B} .10$ Although the previous values were calculated within relatively small radii (e.g., muffin-tin spheres), 10 while the present ones are calculated for the magnetic ions with extended orbitals, it is not surprising that the Fe moments (nearly $75 \%$ spinpolarized) are almost identical because of the strong localization of the Fe $3 d$ orbitals. The present larger Re moments (about 50\% spin-polarized) are ascribed to the delocalized Re $5 d$ orbital behavior.

A high Re-O (and Fe-O) covalent charge density is evident in Fig. 2. In addition, the up-spin Fe charge density is nearly spherical, which corresponds to the formal fullfilled up-spin $\mathrm{Fe}^{3+} 3 d$ orbital; while the down-spin $\mathrm{Re}$ (and $\mathrm{Fe}$ ) density is obviously of the $t_{2 g}$ orbital-like distribution. Moreover, the Re-Re interstitial densities visually arising from the $\mathrm{O} 2 p$ contribution almost have no difference between the up- and down-spin channels, which indicates that the net down-spin Re charge scarcely serves to the Re-Re interaction. In other word, the direct Re-Re interaction due to the itinerant down-spin Re $t_{2 g}$ electrons is nearly impossible. Furthermore, the charge densities in the Re-O-Fe 
bond are quite larger than the Re-Re interstitial densities. A combination of these results with the DOS shown in Fig. 1 leads to a suggestion that the down-spin $\operatorname{Re} t_{2 g}$-O $2 p_{\pi}$-Fe $t_{2 g} p d d-\pi$ coupling rather than the direct Re-Re interaction is responsible for the FiM and HM character of BFRO and SFRO.

In the calculated FiM and HM state of CFRO, however, the down-spin Fe $t_{2 g}$ component decreases at $E_{F}$ due to bent Fe-O-Re bond angles. The reduced Fe-O-Re $p d d-\pi$ coupling, as well as a little stronger site-disorder effect than in BFRO,11 could partly account for the nonmetallicity of CFRO. While a finite down-spin Re $e_{g}$-O $2 p_{\sigma}$-Fe $e_{g}$ $p d d-\sigma$ coupling emerges at $E_{F}$ in CFRO, which is due to the $t_{2 g}-e_{g}$ mixing caused by the lattice distortion. The presence of such a coupling could stabilize the FiM state of CFRO and leads to its increasing $T_{\mathrm{C}}$ as compared with BFRO 11 However, the reasons for the nonmetallicity but high $T_{\mathrm{C}}$ of CFRO are not yet definitely clear.

The $U$ correction lowers the occupied up-spin Fe $3 d$ bands significantly and makes them much narrower and thus behave like a localized $S=5 / 2$ spin. The O $2 p$ states undergo a noticeable change, while the Re $5 d$ bands change insignificantly due to a weak electron correlation. The present $\mathrm{LSDA}+U$ results of SFRO are well comparable with those lately reported with a close value $U_{\text {eff }}=4 \mathrm{eV}$ for Fe. The HM character remains unchanged, and particularly, the DOS at $E_{F}\left[N\left(E_{F}\right)\right]$ caused mostly by the down-spin Re $t_{2 g^{-}} \mathrm{O} 2 p$ hybridized states keeps the value of about 4 states/eV per formula unit (nearly twice as large as that of SFMO1르) as the above LSDA result, since the Re species are not strongly correlated ones. The LSDA $+U$ calculations increase the Fe $(\mathrm{Re})$ spin moments up to $4.16(-1.22) \mu_{B}$ in BFRO, $4.22(-1.27) \mu_{B}$ in SFRO, and $4.29(-1.33) \mu_{B}$ in CFRO. The increases of the Fe spin moments are nearly twice as large as those of the Re moments, due to a stronger localization and electron correlation of the Fe ions. In addition, the 
increasing difference between the present and previous LSDA $+U$ Re spin moments is ascribed to the $U=1 \mathrm{eV}$ correction to the Re $5 d$ states which is adopted in the present calculations but not in previous ones. 10

Now we turn to $\mathrm{Sr}_{2} M \mathrm{MoO}_{6}(M=\mathrm{Cr}, \mathrm{Mn}, \mathrm{Fe}, \mathrm{Co})$. First, the LSDA results of SFMO (see Fig. 3) are in good agreement with the previous ones.10 Like the above case of SFRO, the present Fe spin moment of $3.78 \mu_{B}$ is almost identical with two independently calculated values of 3.79 and $3.73 \mu_{B}, 1$ and the Re spin moment of $-0.44 \mu_{B}$ is larger than two corresponding ones of -0.291 and $-0.30 \mu_{B} .10$ These Re moments are all less spin-polarized $(<45 \%)$. These results suggest once again that the Fe spin is strongly localized while the Mo spin is delocalized. In SFMO the itinerant down-spin Mo $t_{2 g}$ electrons mediate a FM coupling between the full Fe up-spins and are responsible for its HM character via the MoO-Fe coupling, like the above cases of BFRO and SFRO. Owing to the $4 d^{1}$ configuration of the formal $\mathrm{Mo}^{5+}$ ions, $N\left(E_{F}\right)(\approx 2$ states/eV per formula unit being consistent with the previous results 10 id ) is reduced by nearly one half, compared with the above cases of BFRO and SFRO with $\mathrm{Re}^{5+} 5 d^{2}$. It can be seen in Fig. 3 that besides a partial filling of the down-spin Mo $t_{2 g}$ orbitals (formally 1/3 occupied), there is a quite large amount of Mo $4 d$ occupations between -8 and $-4 \mathrm{eV}$ due to strong $p d$ covalence effects. The Mo ions actually have a configuration nearly $4 d^{3.4}$ (see Table I).

For the case of $\mathrm{Sr}_{2} \mathrm{CrMoO}_{6}$, owing to a shift up of the $\mathrm{Cr}^{3+} 3 d$ levels compared with the $\mathrm{Fe}^{3+}$ case of SFMO, the down-spin Mo $t_{2 g}-\mathrm{O} p_{\pi}$-Fe $t_{2 g}$ coupling is reduced around $E_{F}$, which could account for its decreasing $T_{\mathrm{C}}$ and nonmetallic behavior $\mathrm{B}$ (the latter as discussed above for CFRO). Besides, the occupied up-spin $\mathrm{Cr}^{3+} t_{2 g}$ levels are very close to $E_{F}$ and near the empty up-spin $\mathrm{Mo}^{5+} t_{2 g}$ ones. Thus the enhanced hybridization between them (also due to the relatively short Cr-O-Mo bond) leads to a broadened bandwidth, 
which makes the up-spin $t_{2 g}$ hybridized bands crossing $E_{F}$ also and partly occupied. The calculated $\mathrm{Cr}(\mathrm{Mo})$ spin moment is $1.67(-0.65) \mu_{B}$, and the total spin moment per formula unit is $1.13 \mu_{B}$, which is increased by the following $U$ correction up to the expected $2 \mu_{B}$ for an ideal FiM state. While an experimental magnetization value of $\approx 0.5 \mu_{B}{ }^{8}$ is much smaller than these theoretical ones. A significant site-disorder effect could account for this large discrepancy, 122 since a minor difference between the ionic radii $\left(\mathrm{Cr}^{3+} 0.615 \AA\right.$, $\mathrm{Mo}^{5+} 0.61 \AA$ 通 most probably leads to a random structure 3 If a pair of antisite $\mathrm{Cr} / \mathrm{Mo}$ ions leads to a decrease of spin moments by $6 \mu_{B}$, according to the analyses in Ref. 22 , the maximal antisite portion is estimated to be $25 \%$. Note that the formal $\mathrm{Mo}^{5+}$ ions take $4 d^{3.34}$ in the LSDA calculation. The Mo spin is larger in $\mathrm{Sr}_{2} \mathrm{CrMoO}_{6}$ than in $\mathrm{SFMO}$, reflecting a weaker itineracy of the Mo $t_{2 g}$ electrons in the former, 24 which qualitatively accords with its nonmetallic behavior and lower $T_{\mathrm{C}}$.

For $\mathrm{Sr}_{2} \mathrm{MnMoO}_{6}$, its lattice constant is larger than SFMO by $\approx 0.12 \AA$ and the Mn-O bond is quite long, $\mathrm{B}$ which indicates that the Mn ion most probably takes a high-spin bivalence state $\left(\mathrm{Mn}^{2+}, S=5 / 2\right)$ with a large ionic radius. Another evidence to support this argument is that there is no Jahn-Teller lattice distortion in $\mathrm{Sr}_{2} \mathrm{MnMoO}_{6}$ 目 as expected for the $e_{g}$ half-filled $\mathrm{Mn}^{3+}$ manganites. Owing to the Mo $4 d-\mathrm{O} 2 p$-Mn $3 d$ hybridizations, however, the broadened up-spin Mn $e_{g}$ bands cross $E_{F}$ and thus become partly occupied, and so do the down-spin $\mathrm{Mo}^{6+} t_{2 g}$ bands, as shown by the present LSDA FM calculation. For $\mathrm{Sr}_{2} \mathrm{CoMoO}_{6}$, the LSDA FM calculation gives a $\mathrm{HM}$ solution, which is caused by the $2 / 3$ filled down-spin $\mathrm{Co}^{2+} t_{2 g}$ orbitals mixed with the $\mathrm{O} 2 p_{\pi}$ and $\mathrm{Mo}^{6+} t_{2 g}$ ones. Experimentally, the $c$-axis Co-O bond is a little elongated in tetragonal $\mathrm{Sr}_{2} \mathrm{CoMoO}_{6}$ by $0.045 \AA$, $⿴ 囗 ⿱ 一 一)$ which lifts the triple-fold degeneracy of the Co $t_{2 g}$ levels and splits them into a lower-energy doublet $(x z / y z)$ and a higher singlet $(x y)$. While only the weak 
distortion seen by the Co $t_{2 g}$ electrons, as shown by the LSDA calculation, is insufficient to open an insulating gap between the doublet and singlet. Instead the electron correlation determines the gap as seen below.

By comparison of the down-spin Mo $t_{2 g}$ level positions near $E_{F}$, it is suggested that Mo takes $\mathrm{Mo}^{5+}$ in $\mathrm{Sr}_{2} \mathrm{CrMoO}_{6}$ and $\mathrm{Sr}_{2} \mathrm{FeMoO}_{6}$ but $\mathrm{Mo}^{6+}$ in $\mathrm{Sr}_{2} \mathrm{MnMoO}_{6}$ and $\mathrm{Sr}_{2} \mathrm{CoMoO}_{6}$. The $\mathrm{Mo}^{6+}$ state in the latter two is also implied by the shrinked Mo-O bond length. 1 While the finite Mo component at $E_{F}$ in $\mathrm{Sr}_{2} \mathrm{MnMoO}_{6}$ and $\mathrm{Sr}_{2} \mathrm{CoMoO}_{6}$ is entirely ascribed to the $p d d$ hybridizations.

As seen in Table I, however, the Mo $4 d$ population varies insignificantly in $\mathrm{Sr}_{2} M \mathrm{MoO}_{6}$ $(M=\mathrm{Cr}, \mathrm{Mn}, \mathrm{Fe}, \mathrm{Co})$ due to strong intrinsic $p d$ covalency effects, and correspondingly, the $M 3 d$ population increases by a step of $\sim 1$, suggesting nearly same valence states of all $M$. In addition, $\mathrm{Sr}$ and $\mathrm{O}$ retain the valence states of approximately +1.8 and -1.4 respectively, for all $M$. These results should be indicative of nearly a uniform valence state combination in $\mathrm{Sr}_{2} M \mathrm{MoO}_{6}$. Thus the present calculations support a previous suggestion that the $M$-independent spectral feature observed in $\mathrm{Sr}_{2} M \mathrm{MoO}_{6}$ at $\sim 2 \mathrm{eV}$ is ascribed to the $\mathrm{O} 2 p$-Mo $4 d$ transition. In addition, the relative spectral intensity can be qualitatively explained in terms of the varying numbers of the empty Mo $t_{2 g}$ states. It can be seen in Fig. 3 that the down-spin Mo $t_{2 g}$ orbitals at $E_{F}$ have nearly the same partial filling in $\mathrm{Sr}_{2} \mathrm{CrMoO}_{6}$ and $\mathrm{Sr}_{2} \mathrm{FeMoO}_{6}$, while they are almost unoccupied in $\mathrm{Sr}_{2} \mathrm{MnMoO}_{6}$ and $\mathrm{Sr}_{2} \mathrm{CoMoO}_{6}$. Moreover, the number of the Mo $t_{2 g}$ holes is larger in $\mathrm{Sr}_{2} \mathrm{CoMoO}_{6}$ than in $\mathrm{Sr}_{2} \mathrm{MnMoO}_{6}$. These results account for nearly the same O $2 p$-Mo $4 d$ spectral feature of $\mathrm{Sr}_{2} \mathrm{CrMoO}_{6}$ and $\mathrm{Sr}_{2} \mathrm{FeMoO}_{6}$, the more intensive one of $\mathrm{Sr}_{2} \mathrm{MnMoO}_{6}$, and the most intensive one of $\mathrm{Sr}_{2} \mathrm{CoMoO}_{6}$.

The LSDA $+U$ calculation causes nearly the same changes for SFMO as for BFRO and 
SFRO. The Fe (Mo) spin moment is increased up to $4.23(-0.60) \mu_{B}$. The present values are larger than the lately reported ones of $3.97(-0.39) \mu_{B}, 10$ and they are in good agreement with experimental ones of $4.2(-0.5) \mu_{B}$. ${ }^{\text {. }}$ For $\mathrm{Sr}_{2} \mathrm{CrMoO}_{6}$, the up-spin $\mathrm{Cr} t_{2 g}$ bands shift downward and scarcely contribute to $N\left(E_{F}\right)$, and the Cr spin moment is increased up to $2.42 \mu_{B}$. The down-spin Mo $t_{2 g}$ orbitals are rather weakly hybridized with the $\mathrm{Cr}$ $t_{2 g}$ ones, and they form conduction bands crossing $E_{F}$, which could be responsible for its relatively low room-temperature resistivity The present calculations are not sufficient to reproduce the nonmetallic behavior of $\mathrm{Ca}_{2} \mathrm{FeReO}_{6}$ and $\mathrm{Sr}_{2} \mathrm{CrMoO}_{6}$, and therefore a better description of their nonmetallicity could resort to the site-disorder effects in them. For $\mathrm{Sr}_{2} \mathrm{MnMoO}_{6}$, the up-spin $\mathrm{Mn} e_{g}$ and down-spin Mo $t_{2 g}$ bands contribute quite little to $N\left(E_{F}\right)$ due to broadened bandwidths caused by the hybridization effects. The Mn spin moment of $4.54 \mu_{B}$ indicates the high-spin $\mathrm{Mn}^{2+}\left(3 d^{5}\right)$ state with $S=5 / 2$, and a minor Mo spin moment of $0.04 \mu_{B}$ suggests the formal $\mathrm{Mo}^{6+}\left(4 d^{0}\right)$ state. This minor but positive Mo spin is induced by a little stronger hybridization between the up-spin Mn $e_{g}$ and Mo $e_{g}$ orbitals. In terms of the $\mathrm{Mn}^{2+} / \mathrm{Mo}^{6+}$ model, an AFM ground state can be interpreted by superexchange interactions 25 via Mn-O-Mo-O-Mn bonds. 3 Correspondingly, the hybridized bandwidths shown in the above FM calculations will be strongly suppressed in the AFM state, thus giving an insulating gap. Thus the AFM insulating ground state of $\mathrm{Sr}_{2} \mathrm{MnMoO}_{6}$ is reasonably explained. For $\mathrm{Sr}_{2} \mathrm{CoMoO}_{6}$, the electron correlation opens an insulating gap between the down-spin Mo $x z / y z$ doublet and $x y$ singlet. The Co spin moment of $2.93 \mu_{B}$ indicates a high-spin $\mathrm{Co}^{2+}\left(3 d^{7}, S=3 / 2\right)$ state. The calculated small and negative $\mathrm{Mo}^{6+}\left(3 d^{0}\right)$ spin moment of $-0.12 \mu_{B}$ is ascribed to the stronger hybridization with the down-spin Co $x z / y z$ doublet. Like the case of $\mathrm{Sr}_{2} \mathrm{MnMoO}_{6}$, this $\mathrm{Co}^{2+} / \mathrm{Mo}^{6+}$ combination also leads to a superexchange-coupled AFM and insulating ground state of 
$\mathrm{Sr}_{2} \mathrm{CoMoO}_{6}$.

\section{CONCLUSION}

A systematic electronic structure study of $A_{2} \mathrm{FeReO}_{6}(A=\mathrm{Ba}, \mathrm{Sr}, \mathrm{Ca})$ and $\mathrm{Sr}_{2} M \mathrm{MoO}_{6}$ ( $M=\mathrm{Cr}, \mathrm{Mn}, \mathrm{Fe}, \mathrm{Co})$ has been performed by employing LSDA and LSDA $+U$ calculations.

(1) It is demonstrated that the HM character of BFRO, SFRO, and SFMO is not caused by the direct $M^{\prime}-M^{\prime}\left(M^{\prime}=\mathrm{Re}\right.$ or Mo) interactions but the indirect $M^{\prime}$-O-Fe-O- $M^{\prime}$ $p d d-\pi$ couplings which are simultaneously responsible for their FiM character.

(2) It is suggested that a finite $p d d-\sigma$ hybridized state being present at $E_{F}$ in distorted CFRO contributes to its increasing $T_{\mathrm{C}}$ value, compared with the case of BFRO.

(3) The reduced $p d d-\pi$ coupling in $\mathrm{CFRO}$ and $\mathrm{Sr}_{2} \mathrm{CrMoO}_{6}$, as well as the site-disorder effect, could account for their nonmetallic behaviors.

(4) The calculated level distributions and spin moments indicate the $M^{3+} / \mathrm{Mo}^{5+}$ state for $\mathrm{Sr}_{2} \mathrm{CrMoO}_{6}$ and $\mathrm{Sr}_{2} \mathrm{FeMoO}_{6}$ but the $M^{2+} / \mathrm{Mo}^{6+}$ state for $\mathrm{Sr}_{2} \mathrm{MnMoO}_{6}$ and $\mathrm{Sr}_{2} \mathrm{CoMoO}_{6}$, in terms of which both the itinerant FiM state of the former two and the superexchange-AFM insulating state of the latter two can be explained.

(5) The population analyses show that $\mathrm{Sr}_{2} M \mathrm{MoO}_{6}$ have nearly the same valence state combinations due to strong intrinsic $p d$ covalency effects, which accounts for the similar O $2 p$-Mo $4 d$ spectral structures observed in them.

The author thanks Y. Kakehashi and A. Yaresko for their discussions. This work was cosponsored by Max Planck Society of Germany and National Natural Science Foundation of China. 


\section{REFERENCES}

${ }^{1}$ K.-I. Kobayashi, T. Kimura, H. Sawada, K. Terakura, and Y. Tokura, Nature (London) 395, 677 (1998).

${ }^{2}$ K.-I. Kobayashi, T. Kimura, Y. Tomioka, H. Sawada, K. Terakura, and Y. Tokura, Phys. Rev. B 59, 11159 (1999).

${ }^{3}$ M. T. Anderson, K. B. Greenwood, G. A. Taylor, and K. R. Poeppelmeier, Prog. Solid St. Chem. 22, 197 (1993).

${ }^{4}$ W. E. Pickett and D. J. Singh, Phys. Rev. B 53, 1146 (1996), and references therein.

${ }^{5}$ C. Zener, Phys. Rev. 82, 403 (1951).

${ }^{6}$ A. J. Millis, P. B. Littlewood, and B. I. Shraiman, Phys. Rev. Lett. 74, 5144 (1995).

${ }^{7}$ G.-m. Zhao, Phys. Rev. B 62, 11639 (2000).

${ }^{8}$ Y. Moritomo, Sh. Xu, A. Machida, T. Akimoto, E. Nishibori, M. Takata, and M. Sakata, Phys. Rev. B 61, R7827 (2000).

${ }^{9}$ Y. Moritomo, Sh. Xu, T. Akimoto, A. Machida, N. Hamada, K. Ohoyama, E. Nishibori, M. Takata, and M. Sakata, Phys. Rev. B 62, 14224 (2000).

10 Z. Fang, K. Terakura, and J. Kanamori, Phys. Rev. B 63, 180407(R) (2001).

${ }^{11}$ J. Gopalakrishnan, A. Chattopadhyay, S. B. Ogale, T. Venkatesan, R. L. Greene, A. J. Millis, K. Ramesha, B. Hannoyer, and G. Marest, Phys. Rev. B 62, 9538 (2000).

${ }^{12}$ P. Hohenberg and W. Kohn, Phys. Rev. 136, B864 (1964); W. Kohn and L.J. Sham, Phys. Rev. 140, A1133 (1965). 
${ }^{13}$ M. Itoh, I. Ohta, and Y. Inaguma, Mater. Sci. Eng., B 41, 55 (1996).

${ }^{14}$ H. Wu, M. C. Qian, and Q. Q. Zheng, J. Phys.: Condens. Matter 11, 209 (1999).

${ }^{15}$ V.I. Anisimov, J. Zaanen, and O. K. Andersen, Phys. Rev. B 44, 943 (1991); Pan Wei and Zheng Qing Qi, Phys. Rev. B 49, 10864 (1994).

${ }^{16}$ U. von Barth and L. Hedin, J. Phys. C 5, 1629 (1972).

${ }^{17}$ W. E. Pickett, S. C. Erwin, and E. C. Ethridge, Phys. Rev. B 58, 1201 (1998).

${ }^{18}$ D. D. Sarma, P. Mahadevan, T. Saha-Dasgupta, S. Ray, and A. Kumar, Phys. Rev. Lett. 85, 2549 (2000).

${ }^{19}$ R. S. Mulliken, J. Chem. Phys. 23, 1833 (1955).

${ }^{20}$ A. E. Bocquet, T. Mizokawa, K. Morikawa, A. Fujimori, S. R. Barman, K. Maiti, D. D. Sarma, Y. Tokura, and M. Onoda, Phys. Rev. B 53, 1161 (1996).

${ }^{21}$ H. Wu and Q. Q. Zheng, Phys. Rev. B 59, 15027 (1999).

${ }^{22}$ Y. Tomioka, T. Okuda, Y. Okimoto, R. Kumai, K.-I. Kobayashi, and Y. Tokura, Phys. Rev. B 61, 422 (2000).

${ }^{23}$ R. D. Shannon, Acta Cryst. A32, 751 (1976).

${ }^{24}$ In the LSDA $+U$ calculation, the hybridization with the almost full-filled up-spin $\mathrm{Cr} t_{2 g}$ orbitals induces a finite positive Mo spin, which partly counteracts the large negative Mo spin caused by the down-spin Mo $t_{2 g}$ orbitals. The Mo $t_{2 g}$ electrons are less itinerant in $\mathrm{Sr}_{2} \mathrm{CrMoO}_{6}$ than in SFMO, which is also indicated by comparison of the down-spin Mo $t_{2 g}$ bandwidths as seen in Fig. 3 . 
${ }^{25}$ P.W. Anderson, Phys. Rev. 115, 2 (1959). 


\section{TABLES}

Table I. $M 3 d$ and $M^{\prime} 4 d$ or $5 d$ population/spin moment (in $\mu_{B}$ ) of $A_{2} \mathrm{FeReO}_{6}$ $(A=\mathrm{Ba}, \mathrm{Sr}, \mathrm{Ca})$ and $\mathrm{Sr}_{2} M \mathrm{MoO}_{6}(M=\mathrm{Cr}, \mathrm{Mn}, \mathrm{Fe}, \mathrm{Co})$ calculated by LSDA and LSDA $+U$.

\begin{tabular}{ccccc}
\hline \hline & & & \multicolumn{2}{c}{ LSDA $+U$} \\
& \multicolumn{2}{c}{ LSDA } & & \multicolumn{2}{c}{$M^{\prime}$} \\
\hline$A=\mathrm{Ba}$ & $5.76 / 3.72$ & $4.46 /-0.99$ & $5.93 / 4.16$ & $4.50 /-1.22$ \\
$\mathrm{Sr}$ & $5.62 / 3.75$ & $4.36 /-1.05$ & $5.87 / 4.22$ & $4.39 /-1.27$ \\
$\mathrm{Ca}$ & $5.56 / 3.76$ & $4.14 /-1.07$ & $5.84 / 4.29$ & $4.18 /-1.33$ \\
$M=\mathrm{Cr}$ & $3.48 / 1.67$ & $3.34 /-0.65$ & $3.49 / 2.42$ & $3.40 /-0.53$ \\
$\mathrm{Mn}$ & $4.62 / 3.94$ & $3.51 /-0.17$ & $4.81 / 4.54$ & $3.54 / 0.04$ \\
$\mathrm{Fe}$ & $5.65 / 3.78$ & $3.38 /-0.44$ & $5.89 / 4.23$ & $3.43 /-0.60$ \\
$\mathrm{Co}$ & $6.85 / 2.64$ & $3.51 /-0.20$ & $6.96 / 2.93$ & $3.56 /-0.12$ \\
\hline \hline
\end{tabular}




\section{FIGURES}

FIG. 1. The LSDA and LSDA $+U$ DOS for the HM FiM state of $A_{2} \mathrm{FeReO}_{6}(A=\mathrm{Ba}, \mathrm{Sr}, \mathrm{Ca})$. The solid (dashed) line denotes the up (down) spin. Fermi level is set at zero.

FIG. 2. Charge density on the (001) plane of $\mathrm{Ba}_{2} \mathrm{FeReO}_{6}$, with contour from 0.005 to 0.045 a.u. ${ }^{-3}$ in a step of 0.005 a.u. $^{-3}$ (dashed line), and from 0.05 to 0.5 a.u. $^{-3}$ in a step of 0.05 a.u. ${ }^{-3}$ (solid line). The Re ions are located at center and at corner, and the Fe ions in the middle of edges. The Re and Fe ions are separated by oxygen.

FIG. 3. The LSDA and LSDA $+U$ DOS for the FiM state of $\operatorname{Sr}_{2} M \mathrm{FeO}_{6}(M=\mathrm{Cr}, \mathrm{Fe})$ and for the assumed FM state of $\mathrm{Sr}_{2} M \mathrm{FeO}_{6}(M=\mathrm{Mn}, \mathrm{Co})$. 
Fig. $1 \mathrm{Wu}$
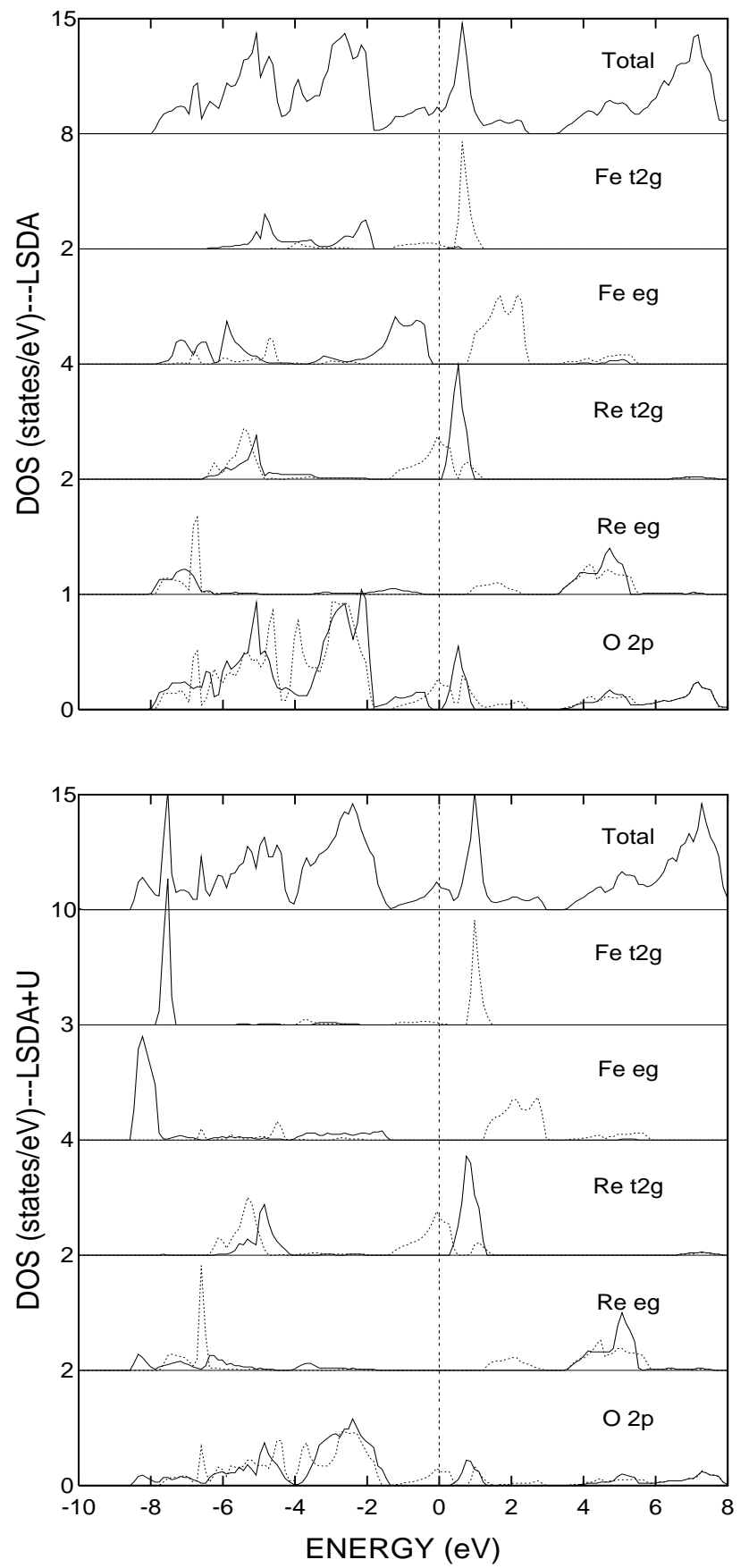

$A=S r$
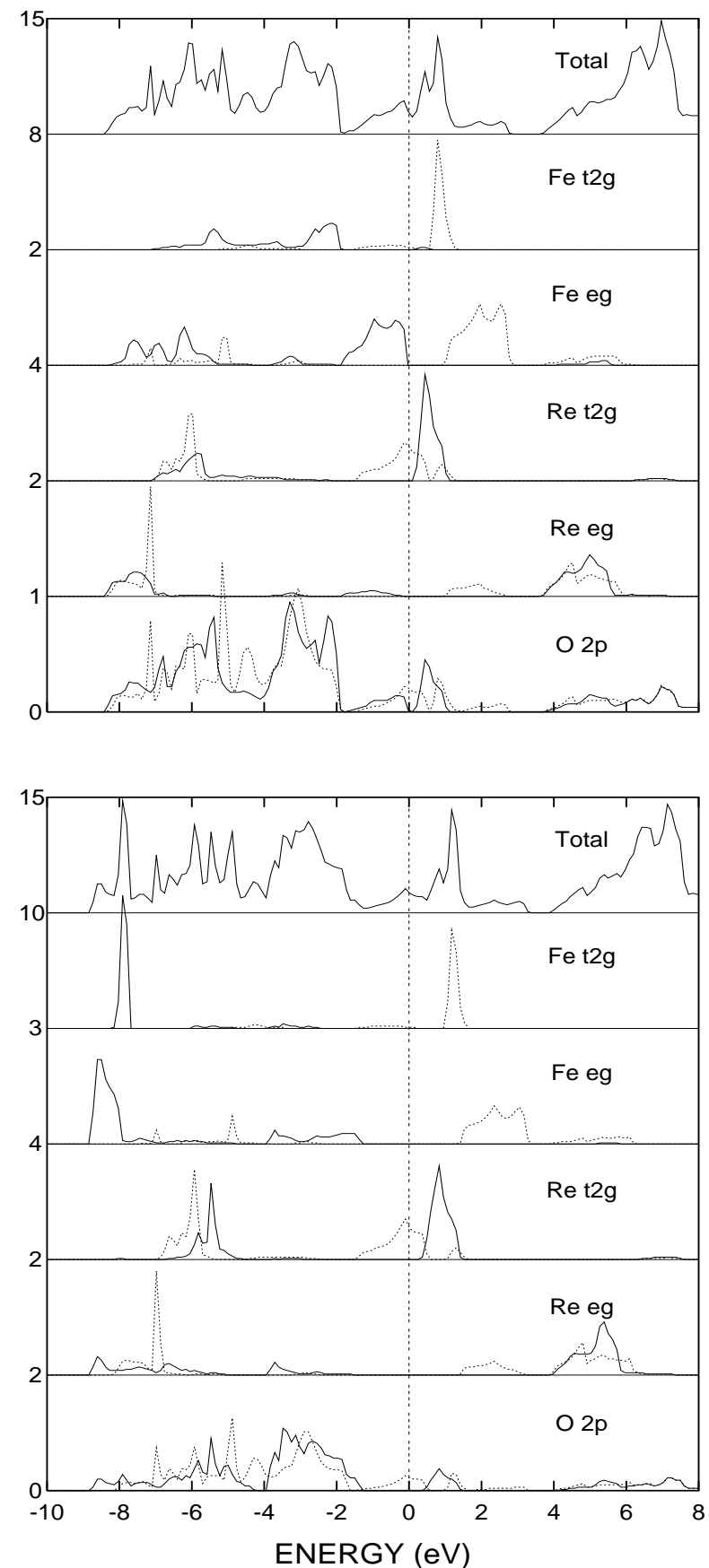

$\mathrm{A}=\mathrm{Ca}$
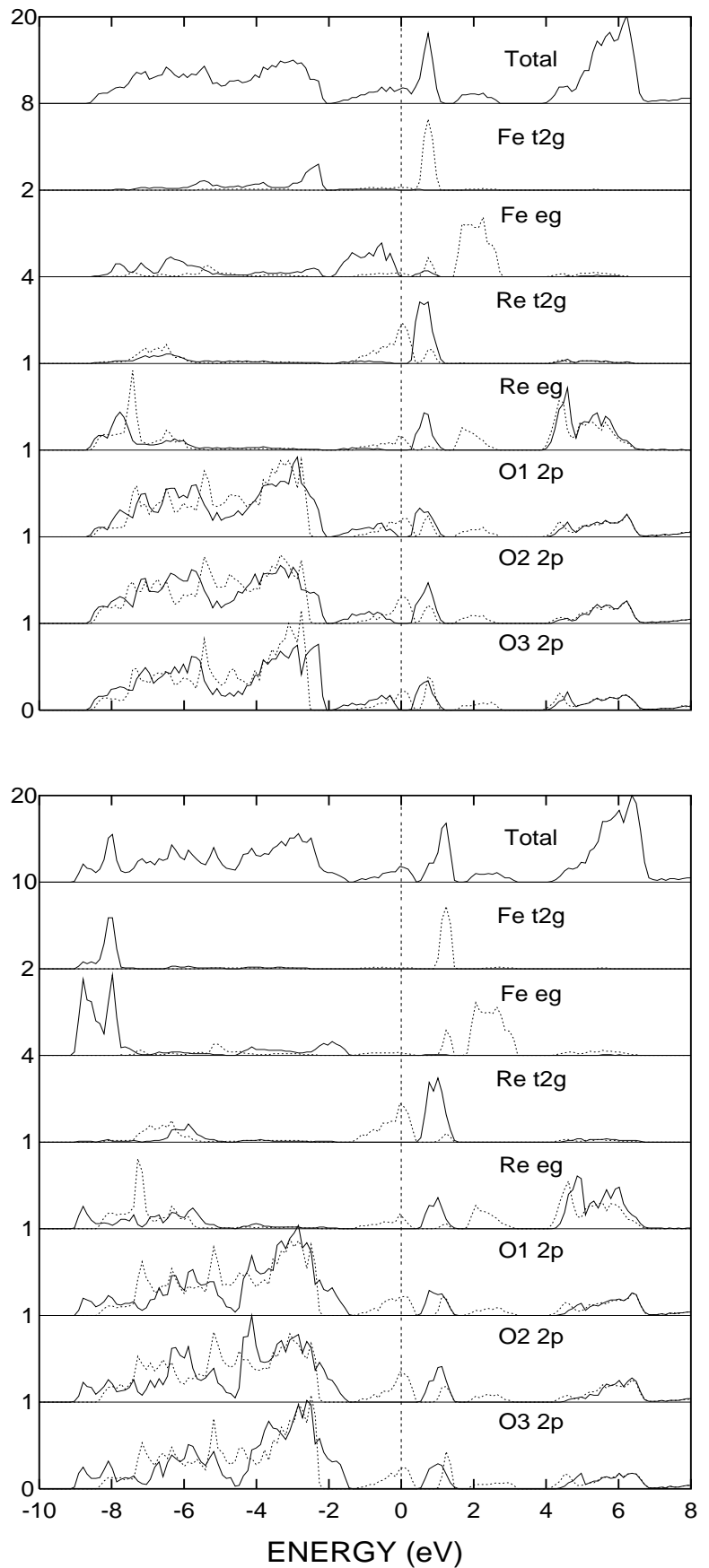

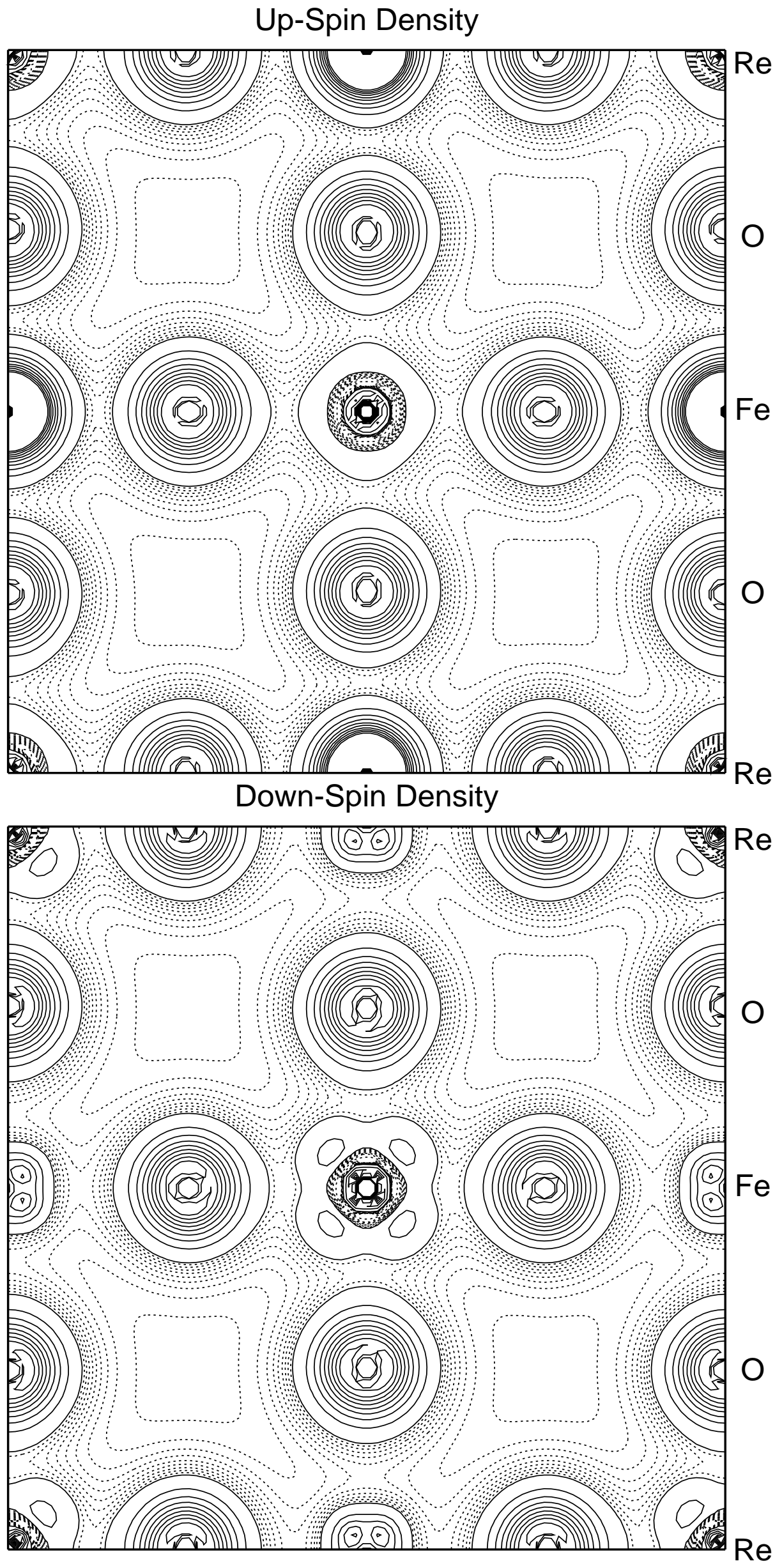
Fig.3 Wu
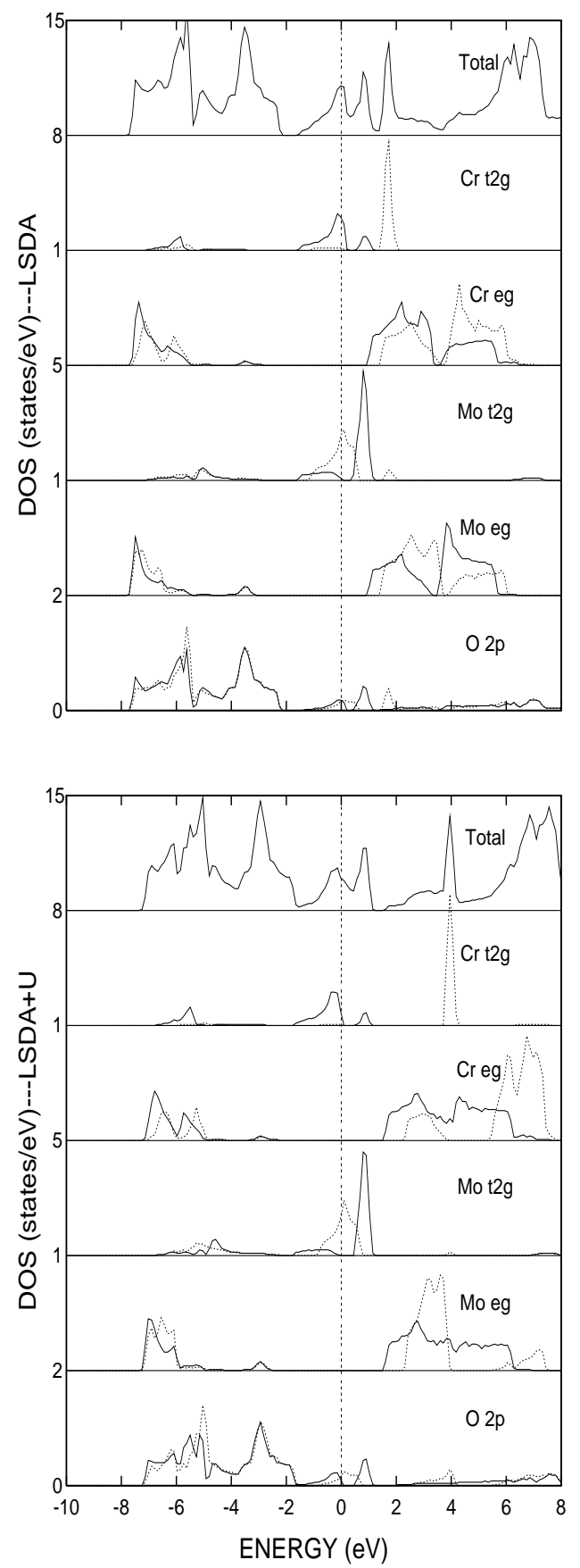

$M=M n$
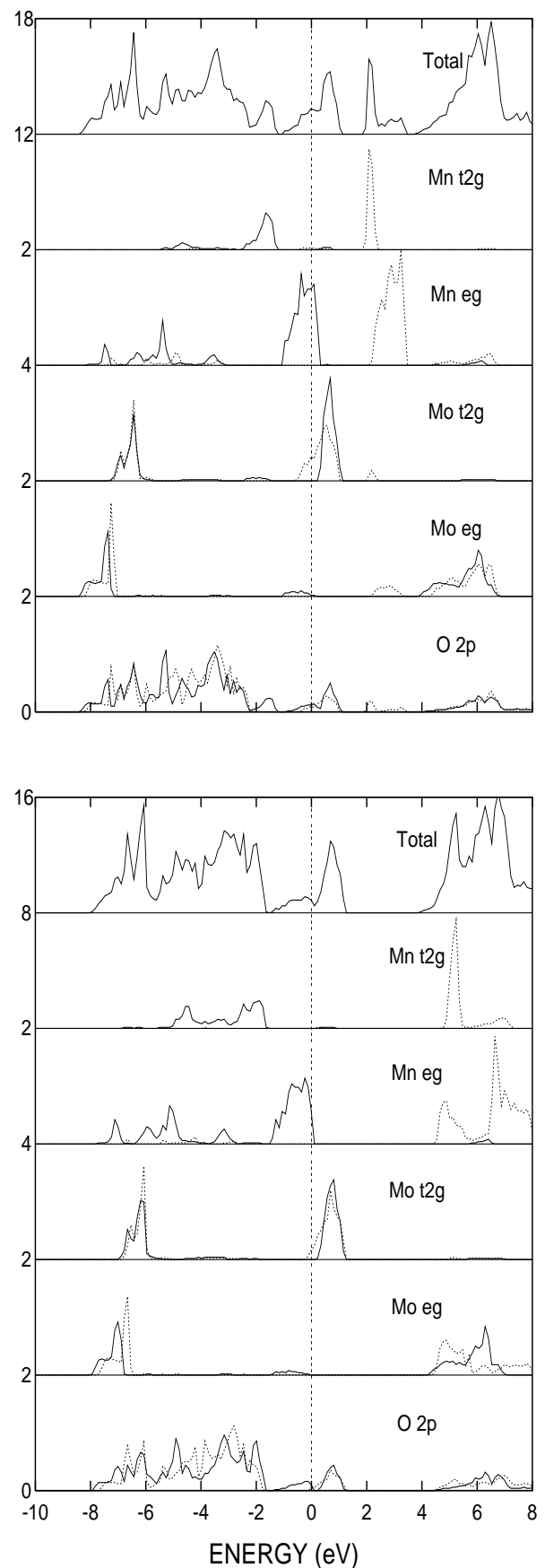

$\mathrm{M}=\mathrm{Fe}$
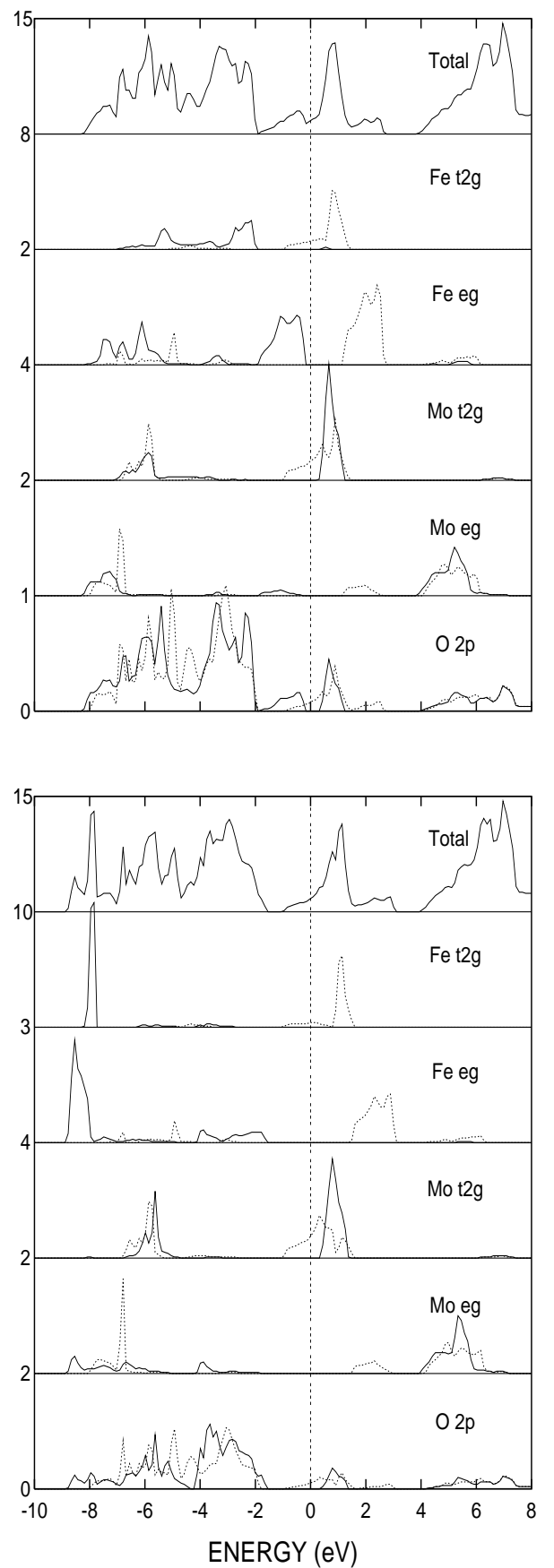

$M=C o$
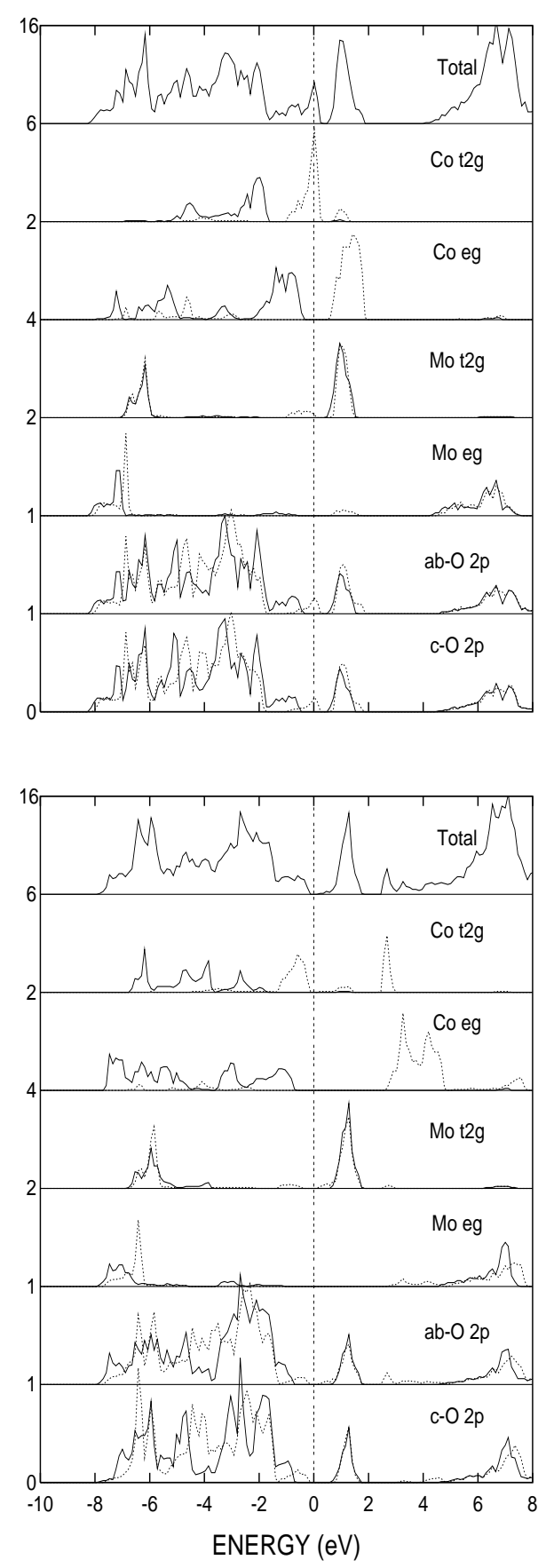